\title{
Occurrence of Beko Disease Caused by Microsporidium seriolae (Microspora) in Hatchery-Reared Juvenile Yellowtail
}

\author{
Motohiko Sano $^{* 1}$, Jun Sato ${ }^{* 2 * 4}$ and Hiroshi Yokoyama*3 \\ *1 Ishigaki Tropical Station, Seikai National Fisheries Research Institute, 148-446 Fukaiota, \\ Ishigaki-shi, Okinawa 907-04, Japan \\ ${ }^{*}$ Goto Station of Japan Sea-Farming Association, Arakawa, Tamanoura-cho, \\ Minamimatuura-gun, Nagasaki 853-05, Japan \\ ${ }^{*}$ Department of Aquatic Bioscience, Graduate School of Agricultural and Life Sciences, The University of Tokyo, \\ Yayoi 1-1-1, Bunkyo-ku, Tokyo 113-8657, Japan \\ ${ }^{* 4}$ Present address: Shibushi Station of Japan Sea-Farming Association, Natsui, Shibushi-cho, \\ Soo-gun, Kagoshima 899-71, Japan
}

(Received November 13, 1997)

\begin{abstract}
Hatchery-produced juvenile yellowtail (Seriola quinqueradiata) were transferred from an indoor tank to sea netpens on June 20, June 30, July 10 and July 31, 1995 (4 groups), and each group was monitored at about 10 day intervals for Microsporidium seriolae, the causative agent of beko disease. Water temperature gradually increased during the study period, ranging from 20.8 to $30.2^{\circ} \mathrm{C}$. In the first 3 fish groups, almost all fish were found to be infected. However, prevalence of infection in the last fish group was much lower than in the other groups. Groups which were transferred later attained their maximum prevalence of infection within a shorter period. Furthermore, we investigated the effects of water temperature and fish size on the development of $M$. seriolae in juvenile yellowtail. Two different fish size classes (average body weight $0.3 \mathrm{~g}$ and $22.0 \mathrm{~g}$ ) were infected with $M$. seriolae to a similar extent. In the histological examination, the development of $M$. seriolae and host recovery were accelerated in fish reared at $25^{\circ} \mathrm{C}$ compared to those kept at $20^{\circ} \mathrm{C}$.
\end{abstract}

Key words: beko disease, Microsporidium seriolae, microsporidian, yellowtail, Seriola quinqueradiata

Beko disease in yellowtail Seriola quinqueradiata, caused by Microsporidium seriolae (Protozoa: Microspora), has often led to retardation in fish growth and even death. Consequently, it has been a major obstacle in seed production of yellowtail in Goto Islands, Kyushu. Microsporidium seriolae forms cyst-like bodies, containing various parasite stages, in the trunk muscle of the host fish. Infected fish show the characteristic clinical sign of a concave body surface, which is the result of liquefaction of muscle tissue around "cysts" (Egusa, 1982). Heavy infection with the parasite emaciates and finally kills the host fish, whereas a lightly infected fish recovers from the disease (Egusa, 1982).

This disease has been often reported from cultured or hatchery-reared yellowtail in Kyushu district, Japan (Egusa, 1982; Yokoyama et al., 1996), but it is not clear whether the disease is limited only to the local area. Artificial infection with $M$. seriolae spores has never been successful and the mode of transmission of this parasite is unknown.
In this study, the occurrence of beko disease and the development of $M$. seriolae in juvenile yellowtail seedlings were investigated in sea netpens. Furthermore, effects of water temperature and fish size on the progress of beko disease were examined.

\section{Materials and Methods}

\section{Seasonal occurrence of beko disease}

Juvenile yellowtail $(0+)$ were bred and reared in indoor tanks supplied with sand-filtered (Tokyo Kyuei Co., Ltd.) sea water at Goto Station of Japan Sea-farming Association in Nagasaki Prefecture. The monitoring site comprised four netpens $(4 \mathrm{~m} \times 4 \mathrm{~m} \times 3.5 \mathrm{~m})$ which were installed to hold the experimental fish in the open sea in front of the station (Fig. 1). Fish were transferred to the sea netpens at four different times: June 20 (G1; average body weight $0.5 \mathrm{~g}, \mathrm{n}=2,000)$, June 30 (G2; 2.1 $\mathrm{g}, \mathrm{n}=2,000)$, July $10(\mathrm{G} 3 ; 7.2 \mathrm{~g}, \mathrm{n}=1,000)$ and July 31 $(\mathrm{G} 4 ; 32.8 \mathrm{~g}, \mathrm{n}=500), 1995$ and maintained there until 
September 11 (G1 and 4) or August 10 (G2 and 3). Unexposed control fish $(n=13,000)$ were reared in an indoor tank ( $8 \mathrm{t}$ ) throughout the study period. Exposed and unexposed fish were fed daily with commercial pellet. Ten days after stocking, 100 fish were removed from the G1 netpen, transferred back to an indoor tank and examined for M. seriolae infection. This was performed to determine whether the infection has occurred prior to the G2 group being transferred to a netpen. Water temperature ranged from 20.8 to $30.2^{\circ} \mathrm{C}$ during the study period.

A hundred fish from each group were randomly sampled at approximately 10 day intervals post-introduction to the netpens and examined for the clinical sign of beko disease. Of the 100 fish, 20 fish were subsequently processed for histological study, 60 were dissected and visually inspected for microsporidian "cysts" in the trunk muscle, and 20 examined microscopically for spores using the Uvitex 2B staining method (Yokoyama et al., 1996). The latter method involved approximately $1 \mathrm{~g}$ of the trunk muscle being taken from each fish. The tissue was then homogenized and smeared on a glass slide, stained with $1 \%$ Uvitex $2 \mathrm{~B}$ in phosphate buffered saline for $10 \mathrm{~min}$, counterstained with $0.5 \%$ Evans blue for $30 \mathrm{sec}$, and observed with a fluorescent microscope (UV excitation). Degree of infection with $M$. seriolae was recorded in the following two types on the basis of intensity of infection: the percentage of fish with an external sign of a concave body surface (Fig. 2) as the prevalence of clinical sign, and the percentage of fish with "cysts" in the trunk musculature (Fig. 3) by gross observation or the percentage of fish with spores in the trunk musculature as the prevalence of infection.

\section{Histological observations}

Two blocks of "cysts" containing muscle tissue from each fish were fixed in $10 \%$ formalin, embedded in paraffin, sectioned at $4 \mu \mathrm{m}$ and stained with hematoxylin and eosin (H \& E) for light microscopy.

The development of $M$. seriolae in the trunk muscle and the associated host reaction were recorded. "Cysts" were categorized into 3 types (I, II, III) for the convenience of the study. Type I (developmental stage): "cysts" contained various parasite stages; these included sporogonal plasmodia, uninucleate stages, sporoblasts and spores (Fig. 4). Type II (maturation stage) : "cysts" do not have sporogonal plasmodia and are filled with mature spores. Most of the "cysts" are disrupted (Fig. 5). Type III (encapsulation stage) : "cysts" are almost

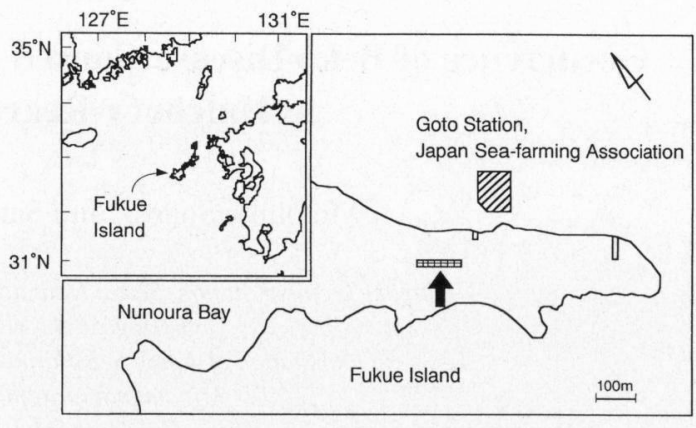

Fig. 1. A map of the monitoring site at Goto Station of Japan Sea-farming Association in Fukue Island, Nagasaki Prefecture. Arrow shows sea netpens to keep the experimental fish.

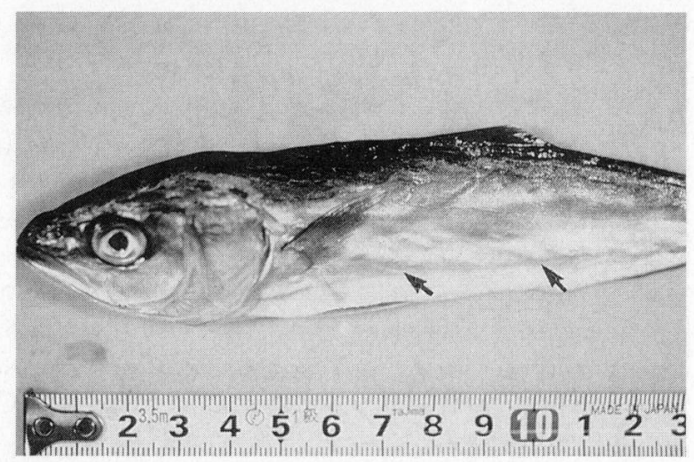

Fig. 2. External sign of beko disease in juvenile yellowtail. Note the concave body surface (arrows).

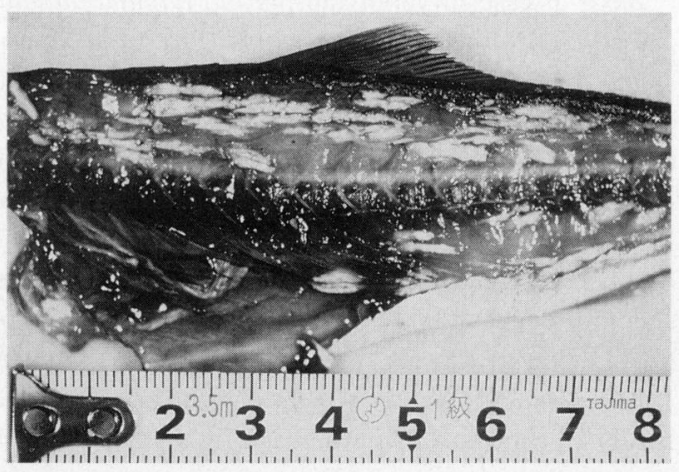

Fig. 3. "Cysts" of Microsporidium seriolae in the trunk musculature of juvenile yellowtail.

completely encapsulated within granulation tissue (Fig. $6)$.

Effect of water temperature on development of $\mathrm{M}$. seriolae 

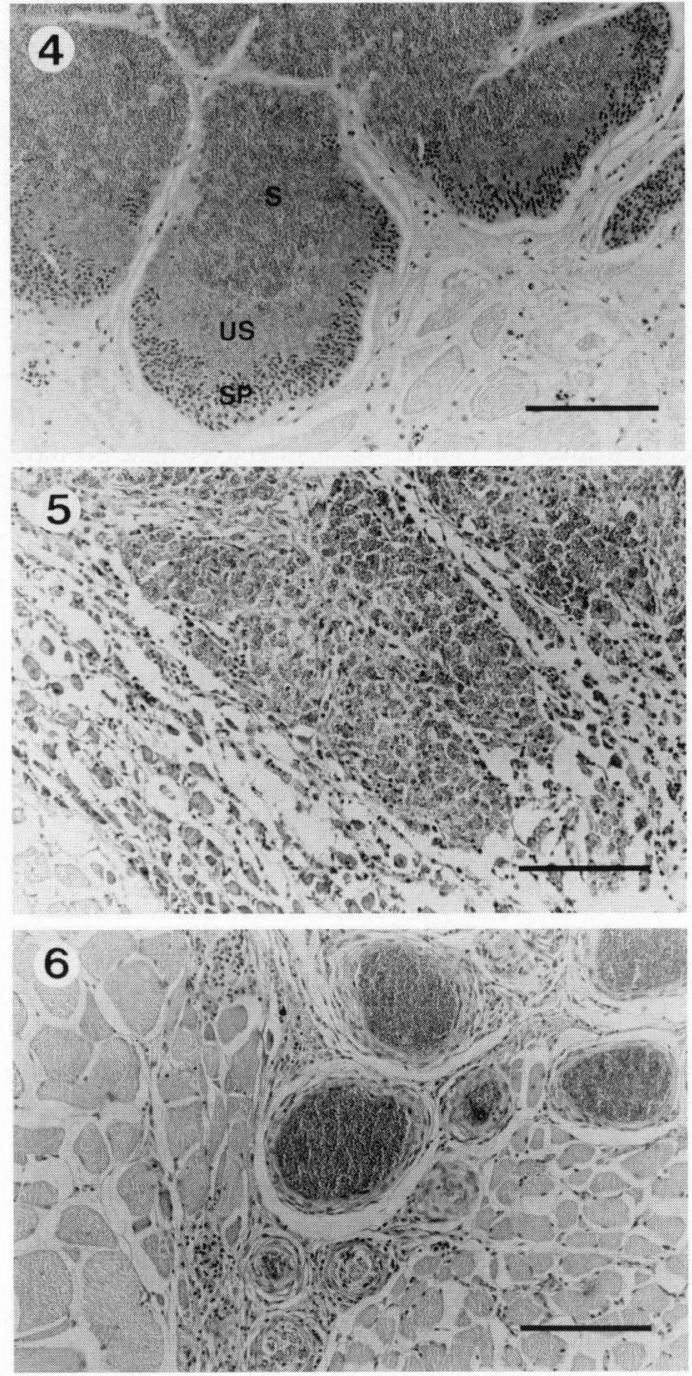

Fig. 4. Developing "cysts" (type I) of M. seriolae in the trunk muscle of yellowtail at 41 days post-transfer. This "cyst" type contains several different parasite stages : sporogonal plasmodia (SP), uninucleate stages (US) and spores $(\mathrm{S}) . \quad \mathrm{H} \& \mathrm{E}$ stain, $\mathrm{bar}=10 \mu \mathrm{m}$.

Fig. 5. Disrupted "cysts" (type II) in the trunk muscle of yellowtail at 51 days post-transfer. The dispersed spores are extensively ingested by phagocytes. Muscle tissue around "cysts" shows diffuse liquefaction and atrophy. Granulation tissue has begun to form. $\mathrm{H}$ $\&$ E stain, bar $=10 \mu \mathrm{m}$.

Fig. 6. Encapsulated "cysts" (type III) in the trunk muscle of yellowtail at 83 days post-transfer with granulation tissues, leading to scar formation. Liquefaction of muscle tissue is not extensive. $\mathrm{H} \& \mathrm{E}$ stain, $\mathrm{bar}=10$ $\mu \mathrm{m}$.
Juvenile yellowtail seedlings $(0+)$ were stocked in a sea netpen for 20 days between May 7 and 27, 1996. They were then divided into two experimental groups of 90 fish (average body weight $7.1 \mathrm{~g}$ ) and transferred back to indoor tanks $(0.5 \mathrm{t})$ supplied with filtered sea water. The two groups were maintained at approximately $20^{\circ} \mathrm{C}$ (average $19.4^{\circ} \mathrm{C}$ ) or $25^{\circ} \mathrm{C}$ (average $24.5^{\circ} \mathrm{C}$ ) for 42 days. After fish were transported to the indoor tanks, fish were randomly sampled from each group at about 10 day intervals, and checked for the clinical sign of beko disease and "cysts" in the trunk musculature by gross observation, and tissues were processed for histological observation.

\section{Effect of fish size on prevalence of $\mathrm{M}$. seriolae}

Two groups of juvenile yellowtail seedlings $(0+)$ (average body weight 0.3 and $22.0 \mathrm{~g}$ ) were reared in sea netpens from July 17 to July 31,1996 . Fish were randomly sampled from each group at about 10 day intervals, and checked for the clinical sign of beko disease and for "cysts" in the trunk musculature by gross observation.

\section{Results}

\section{Seasonal occurrence of beko disease}

Among exposed fish in the sea netpens, moderate mortalities $(26.4 \%$ in $\mathrm{G} 1,36.6 \%$ in $\mathrm{G} 2,15.3 \%$ in $\mathrm{G} 3$, and $2.0 \%$ in G4) were observed. Bacterial infections such as Enterococcus seriolicida and Pasteurella piscicida were noted as well as $M$. seriolae, and the cause of deaths could not be determined.

In $\mathrm{G} 1$, the external symptoms were first noticed at 30 days post-transfer (June 20). Prevalence of clinical sign and prevalence of infection determined by gross observation of "cysts" and Uvitex 2B-staining for spores reached the maximum of $59 \%, 95 \%$ and $100 \%$, respectively, at 50 days post-transfer (Fig. 7). With each subsequent sampling, the fish appeared to gradually recover, i.e. the clinical sign was recorded progressively less frequently. However, prevalence of infection did not decrease proportionately.

The fish transferred back to an indoor tank after 10 days in the G1 group were observed to demonstrate prevalence of clinical sign with a similar rate $(27 \%)$ to those from $\mathrm{G} 1$ at 30 days post-transfer. This result revealed that the $M$. seriolae infection in G1 occurred prior to G2 being transferred to the sea netpen (10 days after G1).

The maximum prevalence of clinical sign and infection in both G2 and G3 were similar to that in G1. 

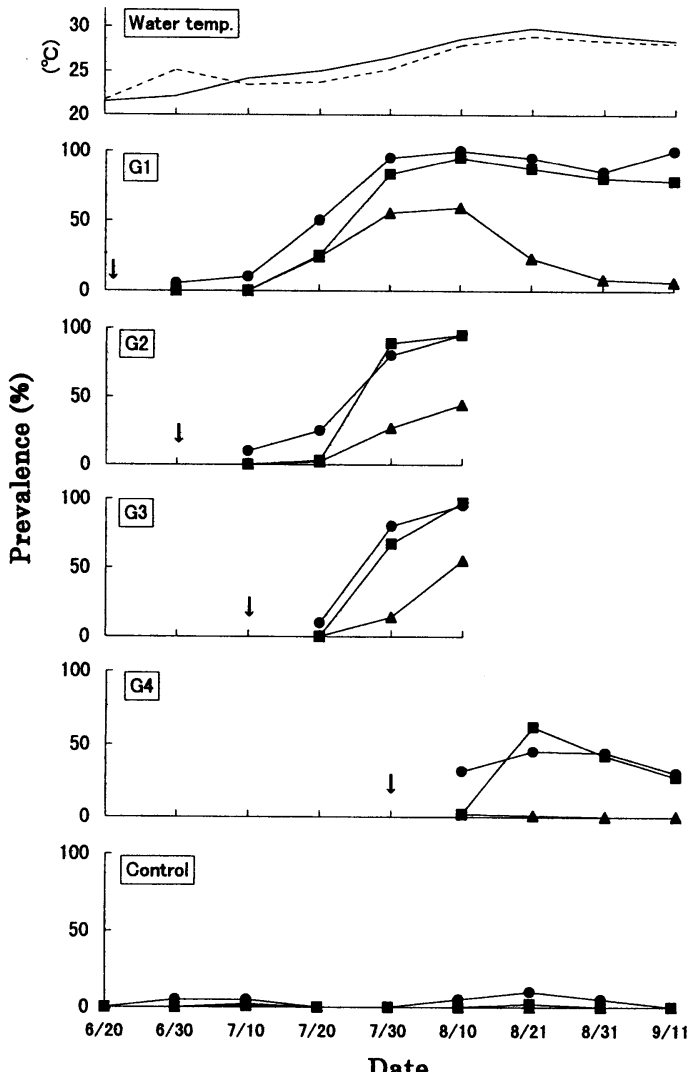

Date

Fig. 7. Prevalence of the clinical sign of beko disease and of infection with $M$. seriolae in experimental groups (G14 and control). Each fish group (G1-4) was transferred from an indoor tank to a sea netpen on the day indicated with an arrow. Prevalence of the clinical sign $(n=100)$ (closed triangles), prevalence of infection by gross observation of "cysts" in trunk muscle of dissected fish $(n=60)$ (closed squares) and by Uvitex 2B-staining for spores of the muscle tissue (n $=20$ ) (closed circles). Each inspection was performed at about 10 day intervals. Water temperature was monitored at $50 \mathrm{~cm}$ below the water surface in the netpen of G1 (a solid line) and indoor tank (a dotted line).

However, groups which were transferred to sea netpens later showed the maximal recorded percentage of the clinical sign and infection within a shorter exposure period (Fig. 7). Prevalence of infection in the last fish group transferred to a sea netpen (G4) was remarkably lower than in the other groups. In the unexposed control group, no clinical sign was observed and only a few fish were found to be infected by gross and microscopic inspection.

\section{Histological observations}

Development of $M$. seriolae progressed more rapidly in fish groups transferred later to sea netpens (Fig. 8). "Cyst" type I could be first observed at 30, 30, 20 days post-transfer in G1, G2 and G3, respectively. The three "cyst" types, I, II and III, were observed successively in each group.

\section{Effect of water temperature}

Prevalence of infection in the fish groups kept in indoor tanks at 20 and $25^{\circ} \mathrm{C}$ reached the maximum recorded level of $55 \%$ at 42 days and $60 \%$ at 20 days, respectively (Fig. 9). In fish maintained at $20^{\circ} \mathrm{C}$, type

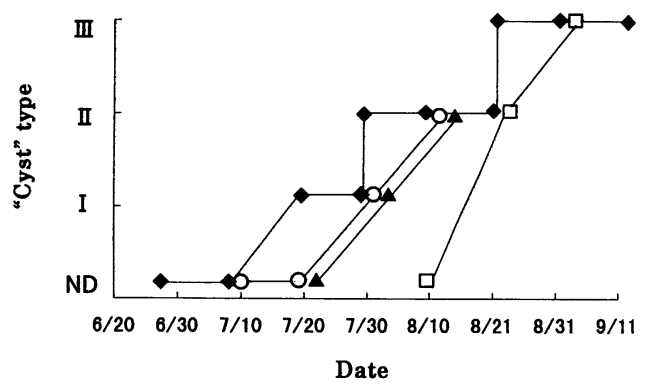

Fig. 8. Observed M. seriolae "cyst" types in the trunk muscle of yellowtail from G1 to G4 with respect to time of post-transfer. Characterization of "cyst" types is described in the text. ND: "cysts" were not detected. G1: closed squares, G2: opened circles, G3: closed triangles, G4: opened squares.

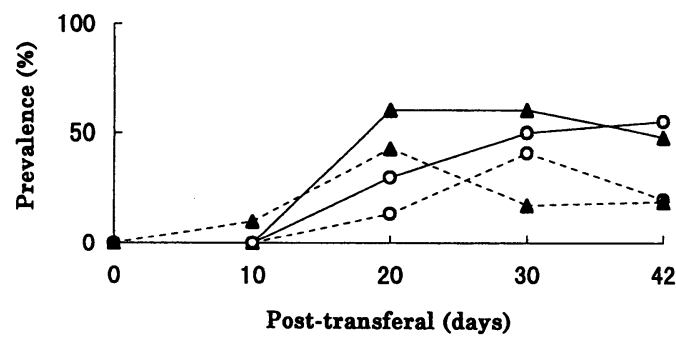

Fig. 9. Prevalence of the clinical sign of beko disease and of infection with $M$. seriolae in fish groups kept at 20 or $25^{\circ} \mathrm{C}$. Fish were stocked in a sea netpen and transferred to an indoor tank, where the water temperature was controlled at 20 or $25^{\circ} \mathrm{C}$. Prevalence of the clinical sign of beko disease (broken line) and of infection (solid line) in the $20^{\circ} \mathrm{C}$ fish group (opened circles) and the $25^{\circ} \mathrm{C}$ fish group (closed triangles). Fish were inspected by gross observation at approximately 10 day intervals. 
I "cysts" were found throughout the study period, and type II were first observed at 42 days post-transfer (Fig. 10). On the other hand, "cyst" types I, II and III on fish kept at $25^{\circ} \mathrm{C}$ were first observed at 20,30 and 42 days post-transfer, respectively.

\section{Effect of fish size}

The clinical sign of beko disease was observed in both fish-size groups. The prevalence of infection recorded at 43 days post-transfer was $64.5 \%$ and $95 \%$ in the smallfish group and the large-fish group, respectively (Fig. $11)$.

\section{Discussion}

This study indicated $M$. seriolae infections were initiated throughout the transfer period from the middle June to late July, though the seasonal pattern in the infectiv-

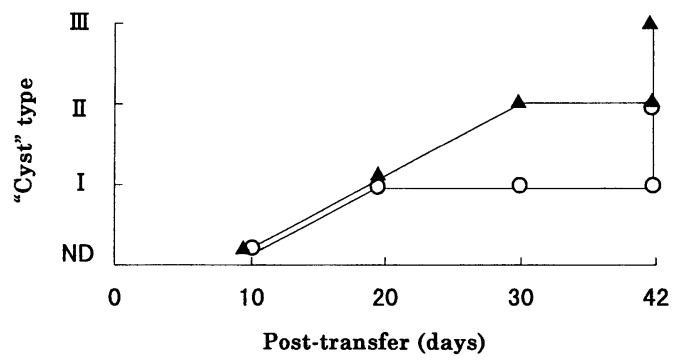

Fig. 10. Observed M. seriolae of "cyst" types in the trunk muscle of yellowtail kept at 20 (opened circles) or $25^{\circ} \mathrm{C}$ (closed triangles) with respect to time posttransferal. Characterization of "cyst" types is described in Figs. 4-6. ND: "cysts" were not detected.

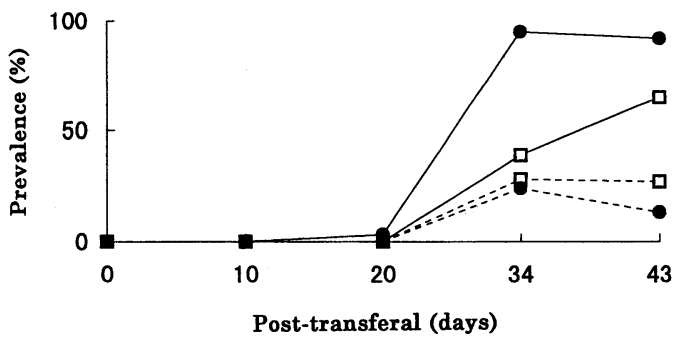

Fig. 11. Prevalence of the clinical sign of beko disease (broken lines) and of infection with $M$. seriolae (solid lines) in the small (average body weight $0.3 \mathrm{~g}$; open squares) or and large-sized ( $22.0 \mathrm{~g}$; closed circles) fish groups reared in sea net-pens. Fish were inspected at approximately 10 day intervals. ity could not be determined. The rate of development of $M$. seriolae varied among the experimental groups (G1-4). Prevalence of infection reached the maximum recorded level within a shorter exposure period in the fish groups transferred later to a sea netpen. This may be based on the time required for the maturation of the parasite following invasion of the trunk muscle, and most likely temperature-dependent, because water temperature gradually increased during the study period. A decrease in the prevalence of clinical sigh in G1 in late August suggested that lightly infected fish could be recovered. Histological observation of scar formation (“cyst" type III) supported this suggestion.

Both prevalences of clinical sign and infection in the last fish group (G4) were remarkably lower than in the other groups. Disease occurrence must be subject to complex interactions between the host (fish size) and the parasite (density of infective agent) under specific environmental influences (water temperature, etc.). No marked difference in the microsporidian infection between the two fish-size groups suggested that the larger fish size of G4 was not the controlling factor. It is probable that the higher water temperature $\left(27-30^{\circ} \mathrm{C}\right)$ during the G4-rearing period might have accelerated the process of parasite's development and host's response. A preliminary observation of less infectivity during midAugust and early November (Takahashi, Maruyama and Yokoyama, unpublished data) may suggest that density of the infective agent decreased during the G4-rearing period. Anyway, in order to clarify parameters involved in the transmission and development of $M$. seriolae, experimental infections which have been successful with many microsporidians (Lom and Dykova, 1992), or in vitro cultivation as has been reported with Heterosporis (= Pleistophora) anguillarum (Kou et al., 1995), are required.

Concerning possible control measures against the beko disease in yellowtail, this study indicates that the sand filtration of sea water is effective in removing the infective agent of $M$. seriolae, although a few fish showing light infection were found in the control group. It has been reported that water temperature influences development of fish microsporidians in the hosts; Microsporidium takedai in salmonid fish (Awakura, 1974), Glugea plecoglossi in ayu (Plecoglosus altivelis) (Takahashi and Egusa, 1977; Takahashi and Ogawa, 1997), Heterosporis (= Pleistophora) anguillarum in eel (Anguilla japonica) (Kanou and Fukui, 1982), and Enterocytozoon salmonis in chinook salmon (Oncorhynchus tshawytscha) (Antonio and Hedrick, 1995). In the present study, we showed 
that the development of $M$. seriolae was accelerated by a water temperature raised from 20 to $25^{\circ} \mathrm{C}$, but could not demonstrate the efficacy of elevated water temperature to control the beko disease. Further investigations are needed to evaluate the potential control of beko disease in yellowtail by elevating water temperature.

The fish reared in a sea netpen for only 10 days from June 20 , when the study was initiated, demonstrated prevalence of the clinical sign to the same extent as G1 which were exposed for 30 days from June 20. Hence, $M$. seriolae infections of G1 occurred within 10 days of exposure. Development of $M$. seriolae was divided into 3 stages by maturation features and the associated host responses, and consequently the types were observed successively in each group. Since the fish were exposed to infection period which continued throughout the transfer period (during mid-June and late July), it seems unlikely that $M$. seriolae invades the fish continuously after the initial infection (within 10 days).

In conclusion, we showed the seasonal occurrence of $M$. seriolae infections and described the developmental processes in hatchery-reared yellowtail juveniles. We also provided additional evidence that elevated water temperature accelerates the developmental rate of $M$. seriolae in yellowtail.

\section{Acknowledgments}

We thank the staff members of Seikai National Fisheries Research Institute and Goto Station of Japan Seafarming Association for the arrangement of this research project. We are grateful to Dr. K. P. Delgahapitiya of the University of Tokyo for useful advice and to Dr. A. Bell of Tokyo University of Fisheries for reviewing this manuscript.

\section{References}

Antonio, D. B. and R. P. Hedrick (1995): Effect of water temperature on infections with the microsporidian Enterocytozoon salmonis in chinook salmon. Dis. Aquat. Org., 22, 233-236.

Awakura, T. (1974): Studies on the microsporidian infection in salmonid fishes. Sci. Rep. Hokkaido Fish Hatchery, 29, 195. (In Japanese.)

Egusa, S. (1982): A microsporidian species from yellowtail juveniles, Seriola quinqueradiata, with "Beko" disease. Fish Pathol., 16, 187-192. (In Japanese.)

Kano, T. and H. Fukui (1982): Studies on Pleistophora infection in eel, Auguilla japonica-I: Experimental induction of microsporidiosis and fumagillin efficacy. Fish Pathol., 16, 193-200. (In Japanese.)

Kou, G.-H., C.-H. Wang, H.-W. Hung, Y.-S. Jang, C.-M. Chou and C.-F. Lo (1995): A cell line (EP-1 cell line) derived from "Beko disease" affected Japanese eel elver (Anguilla japonica) persistently infected with Pleistophora anguillarum. Aquaculture, 132, 161-173.

Lom, J. and I. Dykova (1992): Protozoan parasites of fishes. Developments in aquaculture and fisheries science 26. Elsevier, Amsterdam. 315 p.

Takahashi, S. and S. Egusa (1977): Studies on Glugea infection of the ayu, Plecoglossus altivelis -III. Effect of water temperature on the development of xenoma of Glugea plecoglossi. Fish Pathol., 11, 195-200. (In Japanese.)

Takahashi, S. and K. Ogawa (1997): Efficacy of elevated water temperature treatment of ayu infected with the microsporidian Glugea plecoglossi. Fish Pathol., 32, 193198.

Yokoyama, H., J.-H. Kim, J. Sato, M. Sano and K. Hirano (1996): Fluorochrome Uvitex 2B stain for detection of the microsporidian causing beko disease of yellowtail and goldstriped amberjack juveniles. Fish Pathol., 31, 99-104. 